\title{
Autologous Anti-EGFRvIII 4SCAR-IgT Cells
}

\author{
National Cancer Institute
}

\section{Source}

National Cancer Institute. Autologous Anti-EGFRvIII 4SCAR-IgT Cells. NCI Thesaurus. Code C150518.

A preparation of autologous T-cells that are genetically modified to express immunoglobulins (Igs) that target the negative immunoregulatory human cell surface receptor programmed cell death protein 1 (PD-1; PDCD1; CD279) and programmed death-ligand 1 (PD-L1; CD274) and are transduced with a replication incompetent, selfinactivating lentiviral vector expressing a fourth generation chimeric antigen receptor (4SCAR) consisting of a single chain variable fragment (scFv) targeting anti-epidermal growth factor receptor variant III (EGFRvIII) that is coupled to the costimulatory signaling domains CD28, CD137, CD27 and the zeta chain of the T-cell receptor (TCR), and is fused with the suicide gene inducible caspase 9 (iCasp9), with potential immunostimulating and antineoplastic activities. Upon administration, autologous antiEGFRvIII 4SCAR-IgT cells are directed to and induce selective toxicity in EGFRvIIIexpressing tumor cells. iCasp9 consists of a human FK506 drug-binding domain with an F36V mutation (FKBP12-F36V) linked to human caspase 9. If the administered T-cells lead to unacceptable side effects, the chemical homodimerizer AP1903 can be administered. AP1903 binds to the drug binding FKBP12-F36V domain and induces activation of caspase 9, which results in the apoptosis of the administered T-cells and enhances safety of this agent. EGFRvIII, a tumor-associated antigen (TAA) encoded by an in-frame deletion of exons 2-7 in the EGFR gene, is overexpressed by a variety of cancer cell types and is not expressed by normal, healthy cells. It plays a key role in tumor cell proliferation, tumor angiogenesis and resistance to both radio- and chemotherapy. CD28, CD137 and CD27, T-cell surface-associated co-stimulatory molecules, are required for full T-cell activation and enhance both proliferation of T-cells and antitumor activity. The anti-PD-1 and anti-PD-L1 antibodies produced by the T-cells (IgT) bind to PD-1, expressed on Tcells, and its lig and PD-L1 expressed on cancer cells, respectively. This inhibits PD-1/PDL1-mediated signaling, prevents T-cell inhibition and exhaustion, enhances T-cell activation within the tumor microenvironment (TME) and results in an enhanced T-cellmediated immune response against and toxicity in the EGFRvIII-expressing tumor cells. 
